\title{
Genetically Engineered Microelectronic Infrared Filters
}

\author{
Tom Cwik and Gerhard Klimeck \\ Jet Propulsion Laboratory \\ California Institute of Technology \\ 4800 Oak Grove Dr. \\ Pasadena Ca, 91109 \\ cwik@jpl.nasa.gov
}

\begin{abstract}
A genetic algorithm is used for design of infrared filters and in the understanding of the material structure of a resonant tunneling diode. These two components are examples of microdevices and nanodevices that can be numerically simulated using fundamental mathematical and physical models. Because the number of parameters that can be used in the design of one of these devices is large, and because experimental exploration of the design space is unfeasible, reliable software models integrated with global optimization methods are examined The genetic algorithm and engineering design codes have been implemented on massively parallel computers to exploit their high performance. Design results are presented for the infrared filter showing new and optimized device design. Results for nanodevices are presented in a companion paper at this workshop.
\end{abstract}

\section{Introduction}

The NASA/JPL goal to reduce payload in future space missions while increasing mission capability demands miniaturization of active and passive sensors, analytical instruments and communication systems among others. Currently, typical system requirements include the detection of particular spectral lines, associated data processing, and communication of the acquired data to other systems. Advances in lithography and deposition methods result in more advanced devices for space application, while the sub-micron resolution currently available opens a vast design space. Though an experimental exploration of this widening design space-searching for optimized performance by repeated fabrication efforts-is unfeasible, it does motivate the development of reliable software design tools. These tools necessitate models based on fundamental physics and mathematics of the device to accurately model effects such as diffraction and scattering in opto-electronic devices, or bandstructure and scattering in heterostructure devices. The software tools must have convenient turn-around times and interfaces that allow effective usage. The first issue is addressed by the application of high-performance computers and the second by the development of graphical user interfaces driven by properly developed data structures. These tools can then be integrated into an optimization environment, and with the available memory capacity and computational speed of high performance parallel platforms, simulation of optimized components can proceed. In this paper, specific applications of the electromagnetic modeling of infrared filters will be presented.

The application involves filters of long-wavelength infrared radiation that are under development for a wide range of applications. Over this band (3-18 $\mu \mathrm{m})$ applications range from spaceborne sensors used in the examination of absorption spectra to ground based handheld digital cameras. Electromagnetic fields that are filtered by periodic structures are modeled by Maxwell's equations and discretized by finite element or integral equation methods. An integrated design involves CAD generation of the microdevice structure, generation of a mesh that will discretize the fields, an accurate solution of the electromagnetic fields, and visualization of the resultant field solution. This suite of tools is then integrated into a parallelized genetic algorithm package for design optimization.

A second application involves the heterostructure device design and involves the choice of material composition, layer thicknesses and doping profiles. A general-purpose quantum-mechanics based onedimensional design and analysis tool is used for modeling. As in the above application, this tool is integrated with the parallelized genetic algorithm package for design optimization. Results of this work will be presented in a companion paper at this workshop.

\section{Optimization Via Genetic Algorithms}

Due to the number of parameters involved in instrument design and the broad classes of design optimization needed, global optimization algorithms are considered. A key consideration in using global methods is that they optimize over a carefully defined set of parameters where each parameter is confined to a limited range. Though local optimization methods can be 
employed, they have been found to be highly dependent on starting points, and are typically more successful when the solution space is a relatively smoothly varying function of the design parameters. In the device models considered in this paper, the solution space is non-linear and can appear to be nearly a discontinuous function of the design parameters over regions of the design space. The local methods deploying gradient searches are then difficult to employ for such multi-parameter optimization.

Genetic algorithm optimization employs stochastic methods modeled on principles of natural selection and evolution of biological systems [1,2]. They are global, multi-parameter and do not require constraints on continuity of the solution space. They have been introduced in electromagnetic design and modeling over the last 5 years [3-6] in the relatively diverse areas of antenna design, filter design and the design of scattering structures. At JPL an effort is underway to advance the capabilities for electromagnetic and electronic structure modeling of select microdevice structures. A genetic algorithm package that is easy and flexible to use is part of this effort.

A genetic algorithm optimization package consists of a sequence of procedures that lead to an optimized result. This sequence is common among all genetic algorithms with variants at each stage [2]:

- Model parameterization and gene encoding.

- Initialization of population.

- Evaluation of fitness function for population.

- Selection of subset of population.

- Reproduction through crossover and mutation.

- Evaluation of fitness function and convergence check.

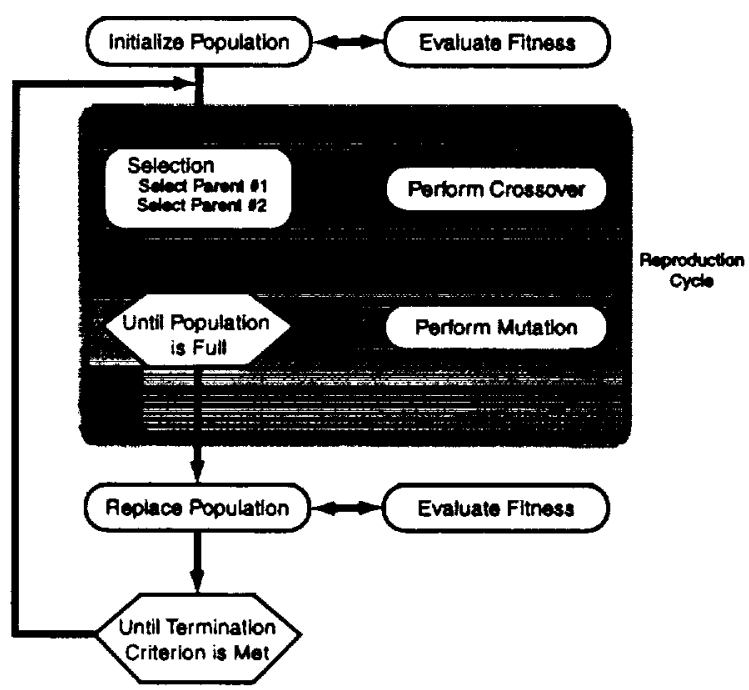

Figure 1. Genetic Algorithm flow diagram (From[5]).
This process is diagrammed in Figure 1 and is the basis for a general package. Because each step in this sequence would require variants dependent on the problem being optimized, a long-term goal is to provide a software framework that is suitable for the select class of design problems considered in this paper. For example, an interface into the range and types of parameters encountered (real-valued device dimension values, integer valued number of layers, or exponent based dopant densities) is to be developed. Similarly an effective means for introducing the fitness function evaluation, i.e. the actual calculation of the electromagnetic or electronic structure result is necessary. This step requires the insertion of large complex codes, typically written in $\mathrm{C}$ or Fortran with associated complex input sets, into the software framework. Ideally there would be little modification of the solver codes when using the genetic algorithm package.

One of the most important stages of the optimization process is the sound parameterization of the design and resultant encoding of the parameterization into a chromosome. The parameters need to be chosen from the design space in an effective manner, limited in range to a set that is physical, and encoded in a meaningful way. For example, the critical device dimensions are typically used, as well as the device material values. If a grid based algorithm such as a finite element solver is used the geometry must be properly parameterized at the CAD stage before a mesh is generated. The geometry should vary in meaningful steps such that known electromagnetic or electronic properties will vary a non-vanishing amount. This variation of geometry or material parameters maps into the stages of population initialization and reproduction through mutation. By allowing variations in the dimensions or materials that produce physically different results, fewer genes need to be stochastically sampled and convergence to an optimum result can occur quicker. Similarly, how the parameter is encoded into a gene is important. For example encoding a real parameter into a binary string or using real-valued encoding is itself a tradeoff [6]. In general, efficient convergence of the optimization algorithm will heavily depend upon the initial parameterization and encoding of the design.

A number of genetic algorithms and packages exist that fit the structure outlined in Figure 1. The key needs for the work in this paper are that the package be flexible enough to allow a range of design parameterizations and be able to exploit high performance computers. First, the different parameterizations and encodings, as well as mutation strategies need to be easily available. Secondly, because the calculations of the fitness function involve electromagnetic and electronic structure calculations that can be quite intensive, executing the genetic algorithm on massively parallel computers is essential for high-fidelity models. These points are encapsulated in PGAPack, a parallel genetic algorithm library [7]. This package consists of a set of library 
routines supplying the user multiple levels of control over the optimization process. The levels vary from default encodings, with simple initialization of parameters and single statement execution, to the ability to modify, at a low-level, all relevant parameters in the optimization process. User written routines for evaluation or crossover and mutation can also be inserted if necessary. The package is written using the Message Passing Interface (MPI) for parallel execution on a number of processors. A master process coordinates the chromosome initialization, selection and reproduction while slave processes calculate the fitness function, including the execution of the electromagnetic or electronic structure code on different processors. For global optimization of realistic structures, the use of high-performance computers is essential to allow designs in a reasonable amount of time. The bottleneck of the optimization process is the electromagnetic or electronic structure code calculation. This result is then used in a calculation of a fitness function (the difference between the calculated and desired result) that is minimized. It is also important that the engineering code be optimized to speed the overall time to design.

\section{Electromagnetic Modeling of Infrared Filters}

Two electromagnetic modeling applications are currently being considered for optimization. The first involves design of multi-bandwidth infrared filters for multi-spectral imagers. The second involves the design of light coupling structures for quantum well infrared photodetectors and uses finite element modeling of Maxwell's equations. This application will not be considered in this paper; the filtering application will be given in detail.

\subsection{Multi-Spectral Filtering}

A multi-bandwidth infrared filter array consists of an array element within a two-dimensional periodic cell. The filter bandwidth, center frequency and polarization properties are dependent upon the shape and size of this element. Different element shapes are used for different applications depending on the design specification. To model a single layer frequency selective surface, a calculation for the induced current on the metallic periodic array deposited on a layered substrate is performed. To model the multiple layering of the design, the scattering parameters due to each screen are cascaded in a separate calculation. The frequency selective surface analysis uses sub-domain basis functions to model the current in a periodic cell. The analysis can be found in [8]. The geometry of a crossed slot periodic surface is shown in Fig. 2. Periodicities are $T_{m}$ and $T_{\eta_{2}}$ in the nonorthogonal $\left(\eta_{1}, \eta_{2}\right)$ coordinate system. Multiple dielectric layers are included below and above the metal slot array.

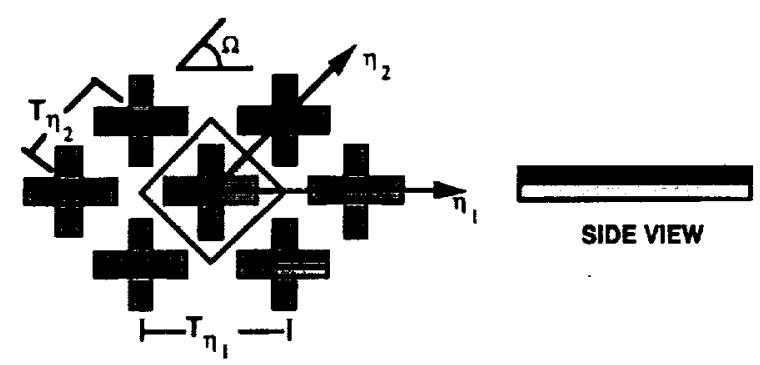

Figure 2. Geometry of crossed-slot frequency selective surface. Plan view and side views are shown.

\subsection{Parameterization and Results}

For the multi-spectral imager application, the goal is to create a filter with a prescribed center wavelength and bandwidth of the transmission response. The filter consists of patterned metal layers fabricated using electron beam lithography. An array of apertures is pattemed into a gold layer deposited on a calcium fluoride substrate. The key parameters of the design are the element shape, periodicities in the $\mathrm{x}$ and $\mathrm{y}$ direction as well as the skew angle $\Omega$, thicknesses of dielectric superstrate and substrate layers, and distance between layers of a sandwich structure.

The fitness function for this application is driven by the requirement of a prescribed center wavelength and bandpass with minimal shoulders outside the bandpass region. A typical response with parameters is shown in Figure 3. The center wavelength, as well as upper and lower edges of the passband is specified. The fitness function specified for this application is a weighted combination of the prescribed center wavelength and a measure of the energy outside the center band. This component is calculated by dividing the area under the square of the transmission curve outside the center band by the total area squared under the transmission response. The goal in this specification is to reduce any energy outside the bandpass region for an optimal multi-band filter design.

The code for the analysis of the frequency selective screen was integrated with PGAPACK and executed on a 256 CPU HP Exemplar X-Class massively parallel processor. For an initial design the center wavelength was chosen to be 4 micrometers, and the bandwidth was minimized outside a region with a $5 \%$ (full) bandwidth. A one millimeter thick calcium floride substrate $(n=1.41)$ was included in the calculation as well as the surface impedance of gold in the near-infrared. A previous design, created without the use of the genetic algorithm optimization package, was used as a 'strawman' to be equaled or bettered. Optimization was carried out 


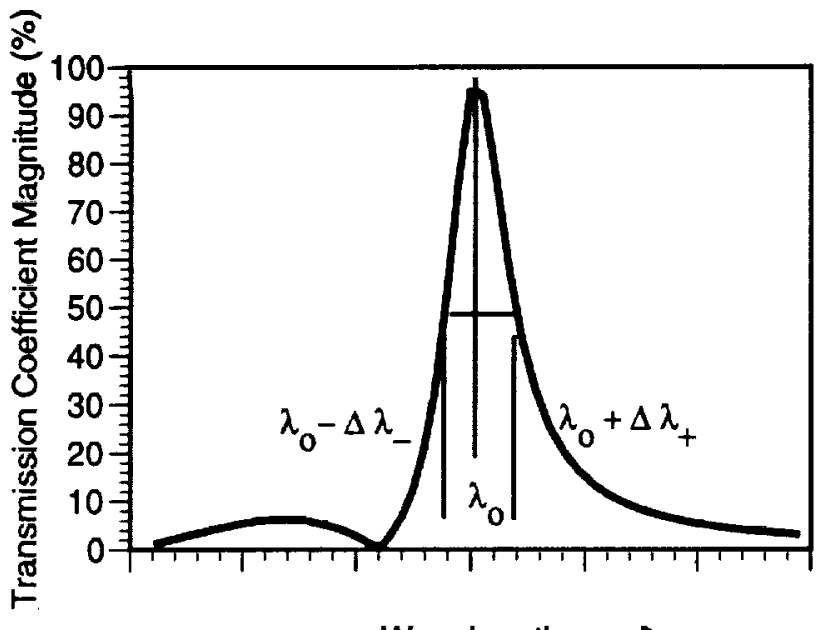

Wavelength

Figure 3. Fitness function design for filter example. Center wavelength and lower and upper bounds of bandpass region are noted.

using the cell size as a parameter and fixing the element geometry to a crossed dipole aperture.

The cell size is encoded as a real parameter using the real encoding funtionality of PGAPack. The initial parameters are randomly selected within a lower and upper bound, and as the real parameters are varied within the genetic algorithm, the parameters are not allowed to evolve outside these bounds. Tournament selection is used to begin reproduction, followed by steady state replacement with a $10 \%$ replacement rate. For the examples considered, two-point crossover is performed with a probability of crossover of 0.85 . The probability of mutation is 0.5 and the allele is fractionally modified. As noted, if the parameter is mutated out of the bounded range it is reset to a value within the range.

Shown in Figure 4 is the resultant design as well as a set of measurements of the fabricated model [9]. For this limited set of optimization parameters, the previous design was equaled but not bettered. The design simulation though was completed with one execution of the genetic algorithm, exhausting the limited set of global parameters needed to minimize the fitness function outlined above. Additional designs with an expanded parameter space are under development. These consist of parameterizing the geometry of the periodic cell into a $N x M$ pixellated grid and allowing each pixel to be metal or no-metal-a binary encoding. In this variation, the geometry can be synthesized to create the prescribed passband. This variation will be presented in a future paper.

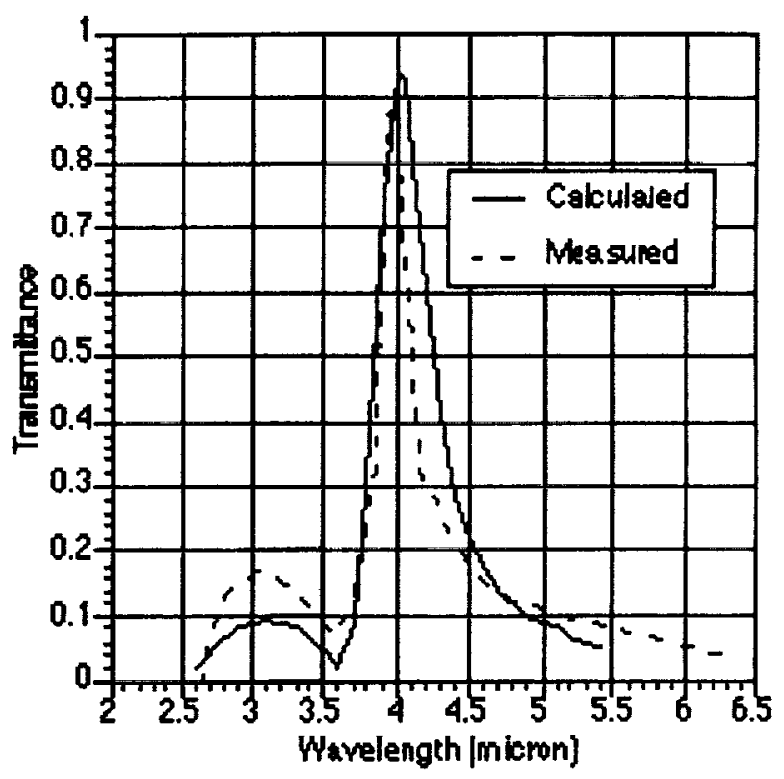

Figure 4. Comparison of numerical simulation and measured transmission spectra for infrared filter

\section{Conclusions}

This paper has examined the use of a genetic algorithm for design of an infrared filter. A versatile genetic algorithm, PGAPACK, has been used for initial designs, optimizing realistic devices over broad design spaces. The optimization package has been integrated with the engineering simulation codes, and executed on parallel computational platforms. These advanced computational resources are necessary if advanced modeling codes are to be used to give high-fidelity simulations of microdevices in the optimization process. Several wall-clock hours on the $64 \mathrm{CPU}$ Exemplar were necessary to achieve the infrared filter design. The proper choice of parameters and proper encoding of these parameters into a chromosome used in the genetic algorithm is also necessary to efficiently reach an optimized solution.

\section{Acknowledgements}

The work described in this publication was carried out by the Jet Propulsion Laboratory, Califomia Institute of Technology under a contract with the National Aeronautics and Space Administration. The supercomputer used in this investigation was provided by funding from the NASA Offices of Earth Science, Aeronautics, and Space Science. Part of the research reported here was performed using HP SPP-2000 operated by the Center for Advanced Computing Research at Caltech; access to this facility was provided by Caltech. 


\section{References}

[1] J. Holland, Adaptation in Natural and Artificial Systems, Ann Arbor, The University of Michigan Press, 1975.

[2] D. Goldberg, Genetic Algorithms in Search, Optimization and Machine Learning, New York, AddisonWesley, 1989.

[3] E. Michielssen, S. Ranjithan and R. Mittra, Optimal Multilayer Filter Design Using Real Coded Genetic Algorithms, Proc. Inst. Elect. Eng., pt. J, vol 139, no.12, pp. $413-420,1992$

[4] R. Haupt, Thinned Arrays Using Genetic Algorithms," IEEE Trans. Antennas Propag, AP-42, 7, pp. 939-999, July 1994.

[5] J. Johnson, and Y. Rahmat-Samii, Genetic Algorithms in Engineering Electromagnetics, IEEE Antennas and Propagation Magazine, Vol 939, No. 4, pp. 7-21, August 1997.

[6] C. Zuffada, and T. Cwik, "Synthesis of Novel All-Dielectric grating Filters Using Genetic Algorithms," IEEE Trans. Antennas Propag, Vol. 46, No. 5, pp.657-663, May. 1998.

[7] G. Levine, Users Guide to the PGAPACK Parallel Genetic Algorithm Library, Argonne Nat. Lab., 95/18, 1996.

[8] T. Cwik T. and R Mittra., Scattering from a periodic array of free-standing arbitrarily shaped perfectly conducting or resistive patches, IEEE Trans. Antennas Propag., vol. AP-35, no. 11, pp. 1226-1233, Nov. 1987.

[9] T. Cwik T. and R. Mittra, The cascade connection of planar periodic surfaces and lossy dielectric layers to form an arbitrary periodic screen, IEEE Trans. Antennas Propag., vol. AP-35, no. 12, pp. 1397-1405, Dec. 1987.

[10] 4. Ksendzov, A., Fernandez, S., Cwik, T., La Baw, C. Maker, P. and Muller' R., "Wedge Filters for Spectral Imaging in the Near IR Using Metal Grids, Proceedings Infrared Astronomical Instrumentation. Vol 3354, 1998.

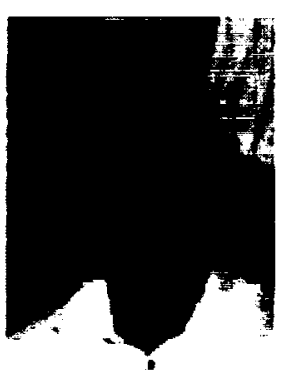

Tom Cwik is supervisor of the High Performance Computing group at the Jet Propulsion Laboratory. Prior to this position he was a Member of Technical Staff in the JPL Microwave Engineering Group, arriving at JPL in 1988. His current research is focused on the application of massively parallel computation for the numerical simulation of electromagnetic systems. This includes work in scattering and light coupling in infrared devices, lenses and filters, and the development of finite element modeling software on distributed memory computers.
Tom Cwik received his BS, MS, and Ph.D. degrees in Electrical Engineering from the University of Illinois, Urbana-Champaign, in 1979, 1981, and 1986, respectively. He has worked at the Very Large Array in Socorro, NM, the National Bureau of Standards in Boulder, $\mathrm{CO}$, and as a Postdoctoral Fellow at the Norwegian Institute of Technology. In 1996 he was appointed an Affiliate Professor in the Department of Electrical Engineering, University of Washington, Seattle, WA.

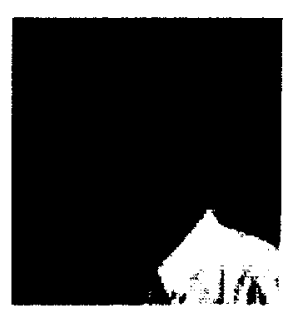

Gerhard Klimeck is a senior technical staff member at the NASA Jet Propulsion Laboratory. His research interest is in the quantum mechanical modeling of electron transport through nanoelectronic devices. Previously he was a member of technical staff at the Applied Research Laboratory of Raytheon (formerly known as the Central Research Lab of Texas Instruments) where he served as manager of the Nanoelectronic Modeling (NEMO) program. The tool is available to the U.S. research community; see http://www.raytheon.com/rtis/nemo/ for details. Dr. Klimeck received his Ph.D. in 1994 from Purdue University where he studied electron transport through quantum dots, resonant tunneling diodes and 2-D electron gases. His research for his German electrical engineering degree, which he obtained in 1990 from Ruhr-University Bochum, concerned the study of laser noise propagation. Dr. Klimeck's work is documented in over 40 publications. He is a member of IEEE, APS, HKN and TBP. 